COMMUNICATIONS IN

ANALYSIS AND GEOMETRY

Volume 10, Number 2, 275-290, 2002

\title{
Immersed Essential Surfaces in Hyperbolic 3-Manifolds
}

TAO LI

\section{Introduction.}

An important question in 3-manifold topology is whether a closed 3-manifold contains $\pi_{1}$-injective surfaces. Embedded $\pi_{1}$-injective surfaces give a lot of information about 3-manifolds, e.g., [15]. But unfortunately, in some sense, most 3-manifolds do not contain embedded $\pi_{1}$-injective surfaces [8]. The main goal of this paper is to prove the contrary to [8] for immersed surfaces, i.e., (in some sense) most 3-manifolds do contain a surface subgroup.

Theorem 1.1. Suppose $X$ is a hyperbolic 3-manifold with boundary a single torus. Then all but finitely many Dehn fillings to $X$ produce 3-manifolds containing $\pi_{1}$-injective surfaces.

This theorem was also proved by Cooper and Long [3] earlier using different methods. The proof that we give here is topological, and an advantage of this approach is that it gives an explicit bound on the number of exceptional surgeries. Theorem 1.1 follows directly from Theorem 1.2 by the deep results in [6], [5]. See below for definitions of $X(\mu)$ and $\Delta(\mu, s)$.

Theorem 1.2. Suppose $X$ is a hyperbolic 3-manifold with boundary a single torus, and $S$ is a two-sided, embedded, incompressible and $\partial$-incompressible surface with boundary slope $s$, and $S$ is not a virtual fiber of $X$. Then there exists a number $\Gamma$ such that $X(\mu)$ contains $\pi_{1}$-injective surfaces for any boundary slope $\mu$ with $\Delta(\mu, s) \geq \Gamma$.

Proof of Theorem 1.1 from Theorem 1.2. It follows from [6], [5] that $X$ contains such incompressible surfaces with at least two distinct boundary slopes arising from the splitting of $\pi_{1}(X)$ associated with the ideal points of certain algebraic curves. Then by Proposition 1.2.7 of [5], the fundamental groups of the splitting surfaces cannot be normal subgroups of $\pi_{1}(X)$, hence they are not virtual fibers and Theorem 1.2 implies Theorem 1.1. 
In this paper, we will mainly prove Theorem 1.2. Unlike [3], we actually do not use the hyperbolic structure. The only thing that we need is that $\pi_{1}(X)$ has no non-peripheral $\mathbb{Z} \oplus \mathbb{Z}$ subgroups, which is equivalent to saying that $\stackrel{\circ}{X}$ has a complete hyperbolic structure by Thurston [14]. Moreover, we will give an explicit bound to the number of exceptional surgeries.

Theorem 1.3. In Theorem 1.2, $\Gamma$ can be chosen to be an explicit linear function of the genus and number of boundary components of $S$.

The idea of the proof is to construct a closed surface from $S$ by connecting pairs of the boundary components of $S$ using long annuli that wind around $\partial X$. By some combinatorial arguments, we show that if both the number of times that the annuli wind around $\partial X$ and the distance between the surgery slope and the slope of $\partial S$ are large, then this closed surface is $\pi_{1}$-injective. Notice that the immersed surface constructed has no triple points.

The techniques in this paper have been used on embedded incompressible surfaces in various papers (e.g., $[12,5])$. The simplicity of the immersion in our construction allows us to apply them to this case. The idea of closing up boundaries of surfaces using long annuli was introduced by B. Freedman and M.H. Freedman in [7], and extensively used in [4, 2, 3].

Notation. Let $\alpha, \beta$ be two slopes on the boundary torus of $X . X(\alpha)$ denotes the closed manifold by Dehn filling along $\alpha$, i.e., by adding a twohandle to $X$ along a simple closed curve with slope $\alpha$ and then capping off the resulting 2-sphere boundary component with a 3-cell. $\Delta(\alpha, \beta)$ denotes the minimal geometric intersection number between two closed curves representing $\alpha$ and $\beta . N(E)$ denotes a small regular neighborhood of $E$, and $|E|$ denotes the number of components of $E$. We use both $\stackrel{\circ}{E}$ and $\operatorname{int}(E)$ to denote the interior of $E$.

Acknowledgments. I would like to thank Dave Gabai for many very helpful conversations, and thank Siddhartha Gadgil and the referee for many comments and corrections.

\section{I-bundle Regions.}

Definition 2.1. Suppose that $M$ is an irreducible 3-manifold with boundary, and $A_{1}, A_{2}, \ldots, A_{s}$ are disjoint annuli in $\partial M$ such that $\partial M-\bigcup_{i=1}^{s} A_{i}$ is incompressible in $M$ and the vertical arcs of each annulus cannot be homotoped rel boundary into $\partial M-\bigcup_{i=1}^{s} A_{i}$. Let $i: D=I \times I \rightarrow M$ be a proper 
map. We call $i$ a product disk of $(M, A)$, if $i(\partial I \times I)$ is a pair of vertical arcs of $A=\bigcup_{i=1}^{s} A_{i}$ and $i(I \times \partial I)$ is a pair of immersed arcs in $\partial M-A$ which cannot be homotoped rel boundary into $A$. We call $\{p\} \times I$ a vertical arc of the product disk for any $p \in I$.

By our definition, any vertical arc of a product disk cannot be homotoped rel boundary into $M-A$.

The following lemma is a simple case of the characteristic pairs in [10,9], see also [11]. For completeness, we give a proof here.

Lemma 2.1. Let $(M, A)$ be as above. Then there is a maximal I-bundle region $J$ in $M$ such that any product disk can be homotoped into $J$.

Proof. First, we will show that there exists such a region $J$ for embedded product disks. Given any two embedded product disks, by the standard cutting and pasting argument, we can assume after isotopy that their intersection is a union of vertical arcs. So, in our proof, we always assume that the intersection of any two embedded product disks is a union of vertical arcs.

We start with $A$ and an embedded product disk $D_{1}$. We thicken them a little to get a small neighborhood of the union of $A$ and $D_{1}$, which is clearly an $I$-bundle. We call it $J_{1}$. Assume that we have constructed $J_{k}$, which is a neighborhood of the union of $D_{1}, D_{2}, \ldots, D_{k}$ and $A$. If there is still an embedded product disk $D_{k+1}$ that cannot be isotoped into $J_{k}$, then we let $J_{k+1}$ be a neighborhood of the union of $D_{1}, \ldots, D_{k+1}$ and $A$. Since $D_{k+1}$ cannot be isotoped into $J_{k}$ such operations increase the Euler characteristic of the non-disk components of $\partial M-J_{k}$. Thus the operations must stop at a certain stage, and we get an $I$-bundle $J^{\prime}$ such that any embedded product disk can be isotoped into $J^{\prime}$.

Furthermore, suppose some component of $\partial M-J^{\prime}$, say $D$, is a disk. Then by the definition of a product disk, each fiber of $J^{\prime}$ cannot be homotoped rel boundary into $\partial M-A$, and hence $D$ together with the fibers of $J^{\prime}$ incident to $\partial D$ is a disk, denoted by $D^{\prime}$. Since $\partial M-A$ is incompressible, $\partial D^{\prime}$ bounds a disk $D^{\prime \prime}$ in $\partial M-A$, and $D^{\prime} \cup D^{\prime \prime}$ bounds a 3-ball because $M$ is irreducible. Therefore, by adding such 3-balls to $J^{\prime}$, we can enlarge $J^{\prime}$ to another $I$ bundle $J$ with canonical fibration such that no component of $\partial M-J$ is a disk. We call $(\partial M-A) \cap J$ the horizontal boundary, which we denote by $\partial_{h} J$, and $\partial J-\partial_{h} J-A$ the vertical boundary of $J$, which we denote by $\partial_{v} J$. Notice that $M-J$ does not contain any embedded product disks with respect to $\left(M-J, \partial_{v} J\right)$. 
Now we show that any product disk can be homotoped into $J$. Let $i: P=I \times I \rightarrow M$ be a product disk. Then $i^{-1}\left(\partial_{v} J\right)$ is a union of disjoint simple arcs and simple closed curves in $P$ since $\partial J$ is embedded in $M$.

If there are simple closed curves in $i^{-1}\left(\partial_{v} J\right)$, we choose an innermost one which bounds a disk $\Delta$ in $P$. Then $i(\partial \Delta)$ is a homotopically trivial curve in the vertical boundary of $J$, and $i(\Delta)$ lies in $J$ (or $M-J$ ). Since $\pi_{2}(M)$ is trivial, we can homotope $i(\Delta)$ to a point on $\partial_{v} J$ and move it out of $J$ (or $M-J$ ) reducing the number of components of $i^{-1}\left(\partial_{v} J\right)$. Hence, we can assume that $i^{-1}\left(\partial_{v} J\right)$ does not contain any simple closed curves.

Since $i(\partial I \times I) \subset A \subset J$, each component of $i^{-1}\left(\partial_{v} J\right)$ is either a vertical arc of $P$ or an arc with both endpoints on the same component of $I \times \partial I$. In the latter case, we choose an outermost such arc, say $\alpha . \alpha$ together with a subarc of $I \times \partial I$, say $\beta$, bounds a disk $\delta$ in $P$. Now $i(\alpha)$ is a $\partial$-parallel arc in $\partial_{v} J$ and, since $\partial M-A$ is incompressible and $M$ is irreducible, $i(\beta)$ is a $\partial$-parallel arc in $\partial_{h} J$ (or $\partial M-J$ ). Since $\pi_{2}(M)$ is trivial, we can homotope $i(\delta)$ out of $J$ (or $M-J$ ) reducing the number of components of $i^{-1}\left(\partial_{v} J\right)$. So we can assume that $i^{-1}\left(\partial_{v} J\right)$ consists of only disjoint vertical arcs in $P$.

If $P$ cannot be homotoped into $J$, then $i^{-1}(M-J)$ is a collection of rectangles of the form $[a, b] \times I$ in $P$, where $[a, b]$ is a subinterval of $I$. $i$ restricted to each of these rectangles is a product disk of $\left(M-J, \partial_{v} J\right)$. By doing some cutting and pasting to $P$ and $\partial_{v} J$, we get an embedded product disk in $M-J$, which contradicts the assumption that $J$ is maximal. Thus any product disk can be homotoped into $J$.

Notation. Let $S$ be an orientable surface and $R \subset S$ be a subsurface of $S$ with $\partial S \subset R$. Let $R^{\prime}=R \cup($ disk components of $S-R$ ), and $\hat{R}=$ $R^{\prime}$ - (disk components of $R^{\prime}$ ).

We define an equivalence relation: $R_{1} \sim R_{2}$, if $\hat{R}_{1}$ and $\hat{R}_{2}$ are isotopic in $S$. Denote the set of surfaces equivalent to $R$ by $[R]$.

Proposition 2.2. Suppose $R_{1}, R_{2}$ are subsurfaces of $S$, and $\partial S \subset R_{1} \cap R_{2}$. Then there exist $R_{1}^{\prime} \in\left[R_{1}\right]$ and $R_{2}^{\prime} \in\left[R_{2}\right]$ such that if a non-trivial curve can be homotoped into each of $R_{1}$ and $R_{2}$, it can be homotoped into $R_{1}^{\prime} \cap R_{2}^{\prime}$.

Proof. If $S$ is a disk or an annulus, then the proof is trivial. So, we can assume that $S$ is a hyperbolic surface with geodesic boundary. For simplicity, we only consider the case that $\hat{R}_{1}$ and $\hat{R}_{2}$ are connected. By our definition, there are no disk components in $S-\hat{R}_{i}$. We isotope $\hat{R}_{1}$ and $\hat{R}_{2}$ to be subsurfaces of $S$ with quasi-geodesic boundaries as follows. If $S-\hat{R}_{i}(i=1$ or 2$)$ contains 
annular components, we isotope $\hat{R}_{i}$ such that the annular components are $\epsilon$ neighborhood of geodesics for some small $\epsilon$. For other boundary components of $\hat{R}_{i}$, we first isotope $\hat{R}_{i}$ so that these boundary components are geodesics, then enlarge $\hat{R}_{i}$ by adding a $2 \epsilon$-neighborhood of the geodesics to it. By choosing $\epsilon$ small enough, we can assume that there is no overlapping of $\hat{R}_{i}$ with itself, $i=1,2$.

For any nontrivial curve of $S$ which can be homotoped into both $R_{1}$ and $R_{2}$, we first homotope it to be a geodesic. It then lies either in both surface constructed above or in an annular component of $S-\hat{R}_{i}$, for some $i$. In the later case we homotope the curve out of the $\epsilon$-neighborhood so that it still remains in the $2 \epsilon$-neighborhood of the geodesics. By our construction, it lies in the intersection of the two surfaces.

Let $R_{1}, R_{2}, R_{1}^{\prime}, R_{2}^{\prime}$ be the surfaces of Proposition 2.2. We denote $\left[R_{1}^{\prime} \cap R_{2}^{\prime}\right]$ by $\left[R_{1}\right] \cap\left[R_{2}\right]$. To simplify our notation, we do not distinguish between $[R]$ and a properly chosen element in $[R]$.

Definition 2.2. Let $X$ be an irreducible 3-manifold whose boundary components are tori, $S$ a two-sided, incompressible, $\partial$-incompressible, embedded surface in $X$, and $M$ be the 3-manifold obtained by cutting $X$ along $S$, i.e., $M \cong X-\stackrel{\circ}{N}(S)$ ( $M$ may not be connected). Let $A=\partial X-\stackrel{\circ}{N}(\partial S)$ be a union of annuli in $\partial M$. We call a map $i: I \times[0, n] \rightarrow X$ an essential rectangle of length $\mathbf{n}$, if $i$ intersects $S$ transversely and $\left.i\right|_{I \times[k, k+1]}$ is a product disk of $(M, A)$ for each $k \in\{0,1, \ldots, n-1\}$.

The following lemma is important to our proof. The same result was proved in [2] for the non-separating case.

Lemma 2.3. Let $X$ be a hyperbolic 3-manifold with boundary a single torus and $S$ be a two-sided, incompressible, $\partial$-incompressible surface in $X$. Suppose $S$ is not a virtual fiber. Then there exists a number $P(S) \in \mathbb{N}$ such that the length of any essential rectangle is less than $P(S)$, where $P(S)=6 g+4 b-6$ and $g, b$ are the genus and the number of boundary components of $S$.

Proof. We assume that $S$ is separating. If not, we can take $S$ together with a parallel copy of $S$ (disconnected) to be our surface.

Let $M_{1}$ and $M_{2}$ be the closures of the two components of $X-S$, and let $M$ be the disjoint union of $M_{1}$ and $M_{2}$. Let $A_{i}=M_{i} \cap \partial X$ for $i=1,2$. Then $\partial M_{1}-A_{1} \cong \partial M_{2}-A_{2}$. 
Let $J_{i}$ be the maximal $I$-bundle region of $\left(M_{i}, A_{i}\right)$ constructed in Lemma 2.1. Let $S_{i}=\overline{J_{i} \cap\left(\partial M_{i}-A_{i}\right)}$ be the horizontal boundary of $J_{i}$ for $i=1,2$. Note that $S_{i}$ and $\partial M_{i}-A_{i}-S_{i}$ have no disk components. We can also assume that $A_{i} \subset J_{i}$. Define $\tau_{i}$ to be an involution of $S_{i}$ such that $\tau_{i}: p_{0} \mapsto p_{1}$, where $p_{0}$ and $p_{1}$ are the endpoints of a fiber of $J_{i}, i=1$ or 2 .

If $J_{i}=M_{i}$ for both $i$, then both $M_{1}$ and $M_{2}$ are $I$-bundles. Hence $\pi_{1}(S)$ is a normal subgroup of $\pi_{1}(X)$ and $S$ is a virtual fiber. So we can assume that $S_{1} \neq \partial M_{1}-A_{1}$. Let $\varphi: \partial M_{1}-A_{1} \rightarrow \partial M_{2}-A_{2}$ be the gluing map, then $X \cong M_{1} \cup M_{2} / x \sim \varphi(x)$. For any $S^{\prime} \in\left[S_{i}\right]$, we can isotope $J_{i}$ so that $J_{i} \cap\left(\partial M_{i}-A_{i}\right)=\hat{S}^{\prime}$ and define $\tau_{i}$ coherently. So we do not distinguish between $\left[S_{i}\right]$ and an element in the equivalent class and always use $J_{i}$ for the coherent $I$-bundle.

Let $T_{1}=\left[S_{1}\right], T_{k}^{\prime}=\tau_{1}\left(\left[S_{1}\right] \cap\left[\varphi^{-1} \circ \tau_{2}\left(\left[S_{2}\right] \cap\left[\varphi\left(T_{k}\right)\right]\right)\right]\right)$, and $T_{k+1}=$ $\left[T_{k}\right] \cap\left[T_{k}^{\prime}\right]$.

Claim. $\left[T_{k}\right] \supsetneqq\left[T_{k+1}\right]$ for any $k$ unless $\left[T_{k}\right]=\left[\partial A_{1}\right]$, where $\left[\partial A_{1}\right]$ is a small neighborhood of $\partial A_{1}$ in $\partial M_{1}-A_{1}$.

Proof of the claim. We have $\left[T_{k}\right] \cong \varphi\left[T_{k}\right] \supseteq\left[S_{2}\right] \cap\left[\varphi\left(T_{k}\right)\right] \cong \varphi^{-1} \circ \tau_{2}\left(\left[S_{2}\right] \cap\right.$ $\left.\left[\varphi\left(T_{k}\right)\right]\right) \supseteq\left[S_{1}\right] \cap\left[\varphi^{-1} \circ \tau_{2}\left(\left[S_{2}\right] \cap\left[\varphi\left(T_{k}\right)\right]\right)\right]=\left[T_{k}^{\prime}\right] \supseteq\left[T_{k}\right] \cap\left[T_{k}^{\prime}\right]=\left[T_{k+1}\right]$. Equalities hold if and only if $\left[\varphi\left(T_{k}\right)\right]=\left[S_{2}\right] \cap\left[\varphi\left(T_{k}\right)\right],\left[\varphi^{-1} \circ \tau_{2}\left(\left[S_{2}\right] \cap\left[\varphi\left(T_{k}\right)\right]\right)\right]=$ $\left[S_{1}\right] \cap\left[\varphi^{-1} \circ \tau_{2}\left(\left[S_{2}\right] \cap\left[\varphi\left(T_{k}\right)\right]\right)\right]$, and $\left[T_{k}^{\prime}\right]=\left[T_{k}\right]$.

If $\left[T_{k+1}\right]=\left[T_{k}\right]$ and $\left[T_{k}\right] \neq\left[\partial A_{1}\right]$ for some $k$, then there exists a boundary component $\gamma$ of $\left[T_{k}\right]$ such that $\gamma$ is not parallel to $\partial A_{1}$. Note that $\gamma$ is a non-trivial curve by our construction. Hence $\varphi(\gamma)$ is a boundary component of $\left[\varphi\left(T_{k}\right)\right]=\left[S_{2}\right] \cap\left[\varphi\left(T_{k}\right)\right]$, and $\gamma_{1}=\tau_{1} \circ \varphi^{-1} \circ \tau_{2} \circ \varphi(\gamma)$ is a boundary component of $\left[T_{k}^{\prime}\right]=\left[T_{k}\right]$.

By our construction, $\delta \cup \tau_{i}(\delta)$ bound an annulus or Möbius band in $J_{i}$ for any simple closed curve $\delta$ in $S_{i}$. Hence, if $\gamma$ is isotopic to $\gamma_{1}$, we can close up the two annuli or Möbius bands bounded by $\varphi(\gamma) \cup \tau_{2} \circ \varphi(\gamma)$ and $\varphi^{-1} \circ \tau_{2} \circ \varphi(\gamma) \cup \gamma_{1}$ to get a torus or Klein bottle in $M$. If $\gamma$ is not isotopic to $\gamma_{1}$ then $\gamma_{2}=\tau_{1} \circ \varphi^{-1} \circ \tau_{2} \circ \varphi\left(\gamma_{1}\right)$ is also a boundary component of $\left[T_{k}\right]$. In this way, we can define $\gamma_{i}$ for any $i$. Since $\left[T_{k}\right]$ has only finitely many boundary components, there exist $i \neq j \in \mathbb{N}$ such that $\gamma_{i}$ is isotopic to $\gamma_{j}$. So we can always close up some annuli or Möbius bands to get an immersed torus or Klein bottle. We shall show that the immersed torus (or Klein bottle) is $\pi_{1}$-injective.

To simplify notation, we do not distinguish between the torus (or Klein bottle) and its image in $X$ under the immersion and denote both by $T$. 
The $\gamma_{i}$ 's are parallel non-trivial curves in $T$ and their images are non-trivial curves in $S$. Since $S$ is two-sided and incompressible, the $\gamma_{i}$ 's are nontrivial in $M$. If the immersion is not $\pi_{1}$-injective, then there is a non-trivial curve, say $\gamma^{\prime}$, in $T$ which intersects each $\gamma_{i}$ non-trivially and is mapped to a trivial curve in $M$. By our construction of $T$, we can homotope $\gamma^{\prime}$ so that it consists of vertical arcs of $J_{1}$ and $J_{2}$. Now that $\gamma^{\prime}$ bounds a disk $D^{\prime}$ in $M$. The pull-back of the intersection of $D^{\prime}$ with $S$ is a collection of simple curves in $D^{\prime}$. By using homotopies as before we can assume that there are no simple closed curves in $D^{\prime}$. We then choose an outermost arc. This arc together with a subarc of $\gamma^{\prime}$ bounds a subdisk in $D^{\prime}$. This subdisk is mapped to either $M_{1}$ or $M_{2}$, which means that the subarc of $\gamma^{\prime}$ (i.e., a vertical arc of $J_{i}$, by assumption) can be homotoped rel boundary into $\partial M_{i}-A_{i}$. This contradicts our assumption on $J_{i}$. Hence the immersion is $\pi_{1}$-injective.

Now we show that $\gamma_{i}$ is not homotopic into $\partial X$. Note that, since $S$ is $\pi_{1}$-injective, any non-trivial and non $\partial$-parallel curve in $\partial M_{h}-A_{h}(h=1$ or 2) is not homotopic into $A_{h}$ in $M_{h}$. Suppose that $\gamma_{i}$ is homotopic into $\partial X$ in $X$. Then there is an immersed annulus $f: E=S^{1} \times[0,1] \rightarrow X$ such that $f$ is transverse to $S, f^{-1}(\partial X)=S^{1} \times\{0\}$, and $f\left(S^{1} \times\{1\}\right) \subset \operatorname{int}\left(M_{1}\right)$ is a curve parallel and close to $\gamma_{i}$. Thus $f^{-1}(S) \cap S^{1} \times\{1\}=\emptyset$. Since $S$ is incompressible and $\partial$-incompressible, by some homotopies, we can get rid of trivial circles in $f^{-1}(S)$ and those arcs in $f^{-1}(S)$ with both endpoints on $S^{1} \times\{0\}$. Hence, we can assume that $f^{-1}(S)$ is a union of disjoint meridian circles in $E$. Since $\gamma_{i}$ is non-trivial and non $\partial$-parallel in $S$, the image of each component of $f^{-1}(S)$ is non-trivial and non $\partial$-parallel in $S$. Let $E_{0}$ be the component of $E-f^{-1}(S)$ that contains $S^{1} \times\{0\}$. Then $\left.f\right|_{E_{0}}$ is an annulus connecting $A_{h}(h=1$ or 2) to a non-trivial and non $\partial$-parallel curve in $\partial M_{h}-A_{h}$, which gives a contradiction. Therefore, we get a $\pi_{1}$-injective and non-peripheral torus in $X$ which contradicts the hypothesis that $X$ is hyperbolic.

It is easy to see that if $\left[T_{k+1}\right] \varsubsetneqq\left[T_{k}\right]$ then there exists a non-trivial simple closed curve $\alpha_{k}$ in $\left[T_{k+1}\right]-\left[T_{k}\right]$. Moreover, we can choose the simple closed curves such that $\alpha_{i}$ is not parallel to $\alpha_{j}$ if $i \neq j$. By an Euler Characteristic argument, there are at most $3 g+2 b-3$ disjoint, essential and non-parallel simple closed curves in $\partial M_{1}-A_{1}$. By our assumption that $S_{1} \neq \partial M_{1}-A_{1}$, there is at least one non-trivial simple closed curve in $\partial M_{1}-A_{1}-S_{1}$. Hence if $k \geq 3 g+2 b-3,\left[T_{k}\right]=\left[\partial A_{1}\right]$. 
Let $i: I \times[0, n] \rightarrow M$ be an essential rectangle of length $n$. Suppose

$$
\begin{aligned}
& \left.i\right|_{I \times[0,1]} \text { is a product disk of }\left(M_{1}, A_{1}\right), \\
& \left.i\right|_{I \times[1,2]} \text { is a product disk of }\left(M_{2}, A_{2}\right), \\
& \left.i\right|_{I \times[2,3]} \text { is a product disk of }\left(M_{1}, A_{1}\right),
\end{aligned}
$$

$$
\begin{array}{ll}
\text { By }(1), & i(I \times\{1\}) \in\left[T_{1}\right], \\
\text { by }(2), & i(I \times\{1\}) \in\left[S_{2}\right] \cap\left[\varphi\left(T_{1}\right)\right], \\
& i(I \times\{2\}) \in \tau_{2}\left(\left[S_{2}\right] \cap\left[\varphi\left(T_{1}\right)\right]\right), \\
\text { by }(3), & i(I \times\{2\}) \in\left[S_{1}\right] \cap \varphi^{-1} \circ \tau_{2}\left(\left[S_{2}\right] \cap\left[\varphi\left(T_{1}\right)\right]\right), \\
& i(I \times\{3\}) \in\left[T_{1}^{\prime}\right] \cap\left[T_{1}\right]=\left[T_{2}\right], \\
& \ldots \ldots
\end{array}
$$

Thus, if $n \geq 2(3 g+2 b-4)+1, i(I \times\{2(3 g+2 b-4)+1\}) \in\left[T_{3 g+2 b-3}\right]=$ $\left[\partial A_{1}\right]$ which contradicts our definition of a product disk.

Hence we have $n \leq 2(3 g+2 b-4)$.

Similarly, if $i(I \times[0,1])$ is a product disk of $\left(M_{2}, A_{2}\right)$ and $J_{2}=M_{2}$ then we get $n \leq 2(3 g+2 b-4)+1$. So in any case, we have

$$
n \leq 6 g+4 b-7 \text {. }
$$

Corollary 2.4. Let $X$ be a hyperbolic 3-manifold with boundary a single torus and $i:(S, \partial S) \rightarrow(X, \partial X)$ a $\pi_{1}$-injective surface. Suppose there is a constant $C \in \mathbb{N}$ such that the genus of $S$ is less than $C$. Then there are only finitely many possible slopes for the boundary circles of $i(\partial S)$.

Proof. Let $S^{\prime}$ be an embedded, two-sided, incompressible, $\partial$-incompressible surface in $X$ and suppose $S^{\prime}$ is not a virtual fiber. Let the boundary slope of $\partial S^{\prime}$ be $s$ and the boundary slope of $i(\partial S)$ be $\mu$. As in the proof of Theorem 1.1 it suffices to show that $\Delta(\mu, s)$ is bounded.

The components of $i^{-1}\left(S^{\prime}\right)$ are disjoint simple arcs or simple closed curves in $S$ because $S^{\prime}$ is embedded. Let $g$ be the genus of $S$ and $b$ be the number of boundary components of $S$. Then $i^{-1}\left(S^{\prime}\right)$ consists of at least 
$\frac{1}{2} b \Delta(\mu, s)$ simple arcs in $S$. By an Euler characteristic argument, there are at most $6 g+3 b-6$ disjoint nonparallel nontrivial simple arcs in $S$. Since $g \leq C$, if $\Delta(\mu, s)$ is large, there are many parallel arcs in $S$ and we get an essential rectangle with large length violating Lemma 2.3. So $\Delta(\mu, s)$ cannot be too large.

\section{Remarks.}

1. In [1] and [13], it was shown that for many 3-manifolds with boundary a torus there are infinitely many boundary slopes realizing $\pi_{1}$-injective surfaces. Corollary 2.4 says that as the boundary slope increases, the genus of the surface increases.

2. Corollary 2.4 is not a deep result. The following elegant argument is due to Dave Gabai. Let $X$ be a hyperbolic 3-manifold with a single cusp and let $S$ be a $\pi_{1}$-injective surface mapping cusps to cusp. Suppose $S$ has the least area in its homotopy class. Then, by Gauss-Bonnet, $\operatorname{Area}(S) \leq$ $-\chi(S)=2 g-2+b$. On the other hand, we let $T$ be a horospherical torus in $X$. Then $S \cap T$ is a union of $b$ closed curves (in $T$ ) of length at least $l$, where $l$ depends on the slope of the closed curves. By hyperbolic geometry, the area of the cusps of $S$ is at least $k b l$, where $k$ is a constant and $b$ is the number of cusps of $S$. Hence we have $k b l \leq \operatorname{Area}(S) \leq 2 g-2+b$. Since $g$ is bounded, $l$ cannot be too large and $S$ can realize only finitely many slopes.

\section{Construction of the injective surfaces.}

Let $X$ be a hyperbolic 3-manifold with boundary a torus and $S$ be a twosided, incompressible, $\partial$-incompressible, embedded surface which is not a virtual fiber. As before, we assume that $S$ is separating, otherwise we take $S$ together with a parallel copy of $S$ (disconnected) to be our surface. For simplicity we only consider the case that $S$ has two boundary components. The proof is similar for the case that $S$ has more than two boundary components.

Let $T^{2} \times I$ be a product neighborhood of $\partial X$ and $S^{\prime}$ be a parallel copy of $S$. We construct our immersed surface $T$ by connecting the two circles of $\partial\left(S^{\prime}-T^{2} \times I\right)$ using an annulus that winds (in $T^{2} \times I$ ) around $\partial X K$ times as shown in Figure 1 (a). Thus $T \cap S$ is a collection of $2 K \partial$-parallel disjoint simple closed curves. We call this annulus the long annulus.

We define a retraction map $\pi: X \rightarrow \overline{X-T^{2} \times I}$ by fixing points in $X-T^{2} \times I$ and mapping every interval $\{p\} \times I$ of $T^{2} \times I$ to the point $(p, 1)$, where $p \in T^{2}$. 
Lemma 3.1. If $K \geq P(S)+1$ then $T$ is $\pi_{1}$-injective in $X$.

Proof. Suppose not, then there exists an immersed closed curve $l$ in $T$ such that $l$ is contractible in $X$ but not contractible in $T$.

Let $p$ be the number of arcs in the intersection of $l$ with the long annulus. Notice that $p \geq 1$ since $S$ is incompressible and two-sided. We homotope $l$ to minimize $p$ and the number of points in its intersection with $S$.

Since $l$ is contractible in $X$, there is a map $j: D \rightarrow X$ such that $j(\partial D)=$ $l$, where $D$ is a disk. We see that $|l \cap S|=2 K p$ and $j^{-1}(S)$ is a collection of disjoint simple arcs in $D$ since $S$ is embedded.

The two circle components of $\partial\left(S^{\prime}-T^{2} \times I\right)$ divides $l$ into $2 p$ subarcs, namely $\alpha_{1}, \beta_{1}, \alpha_{2}, \beta_{2}, \ldots, \alpha_{p}, \beta_{p}$, where $\bigcup_{i=1}^{p}\left(\alpha_{i} \cup \beta_{i}\right)=\partial D, j\left(\beta_{i}\right)$ is a subarc of $l$ lying entirely in the long annulus and $j\left(\alpha_{i}\right)$ is a subarc of $l$ lying entirely in $S^{\prime}$. Thus $j^{-1}(S) \cap \partial D \subset \bigcup_{i=1}^{p} \beta_{i}$ and $\left|j^{-1}(S) \cap \beta_{i}\right|=2 K$ for each $i$. We call the $\alpha_{i}$ 's $\alpha$-arcs, and the $\beta_{i}$ 's $\beta$-arcs. These $\alpha$-arcs and $\beta$-arcs appear on $\partial D$ alternately.

Claim 1. There are no $\operatorname{arcs}$ in $j^{-1}(S)$ whose endpoints are both in the same $\beta_{i}$.

Proof of Claim 1. Suppose there are such arcs. We choose an outermost one, say $\gamma$, then $\gamma$ together with a subarc $\hat{\beta}$ of $\beta_{i}$ bounds a bigon in $D$. Hence $\pi \circ j(\gamma)$ must be a $\partial$-parallel arc in $\pi(S)$. Since $\gamma$ is outermost and $S$ is $\partial$-incompressible, both endpoints of $j(\hat{\beta})$ must lie in the same component of $T \cap S$ and $j(\operatorname{int}(\hat{\beta})) \cap S=\emptyset$. So we can homotope $l$ to have fewer points of intersection with $S$, which contradicts our assumption. This proves Claim 1 .

We call an arc in $j^{-1}(S)$ a long arc if it cuts $D$ into two components such that each of them contains at least two $\alpha$-arcs.

Claim 2. There exists a $k \in \mathbb{N}$ such that the endpoints of no long arc lie in $\beta_{k}$.

Proof of Claim 2. Consider all the long $\operatorname{arcs}$ of $j^{-1}(S)$ in $D$ and choose an outermost one. This together with a subarc of $\partial D$ bounds a bigon that does not contain long arcs. Suppose the bigon contains arcs $\alpha_{k}$ and $\alpha_{k+1}$, then $\beta_{k}$ is as needed because $j^{-1}(S)$ consists of disjoint simple arcs. This proves Claim 2.

Consider all the arcs with an endpoint in $\beta_{k}\left(\beta_{k}\right.$ as in claim 2). By claim 1 , the other endpoint of such an arc must lie in either $\beta_{k-1}$ or $\beta_{k+1}$. Since $\left|\beta_{k} \cap j^{-1}(S)\right|=2 K$, we have at least $K$ parallel arcs which are all parallel to $\alpha_{k}$ (or $\alpha_{k+1}$ ), as shown in Figure 1 (b). 


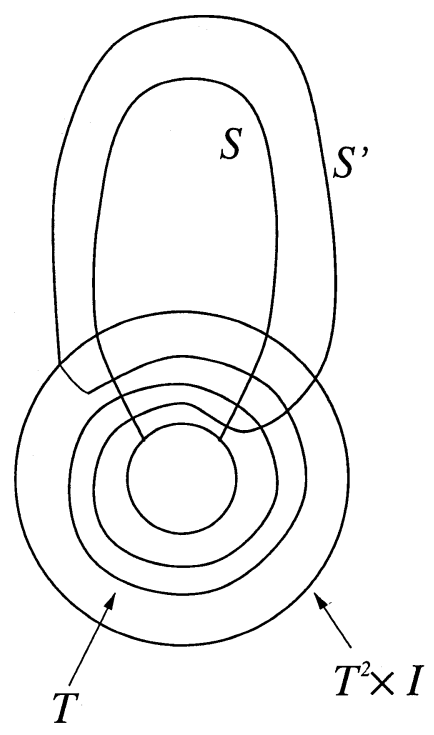

(a)

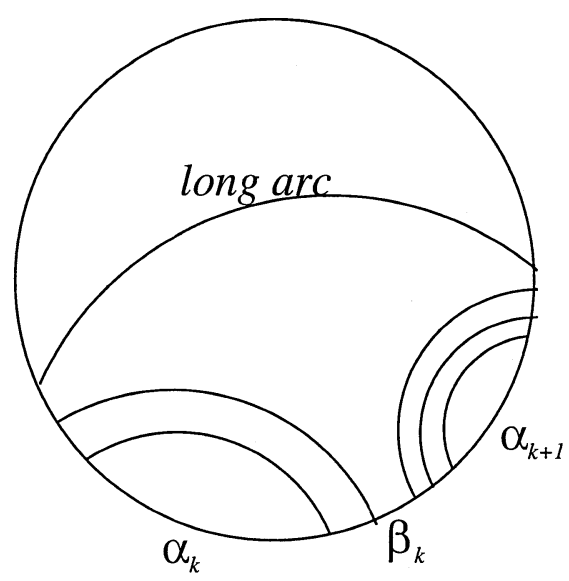

(b)

Figure 1.

Notice that $\pi \circ j\left(\alpha_{k}\right)$ is not a $\partial$-parallel arc in $\pi\left(S^{\prime}\right)$, otherwise we can homotope $l$ to have fewer points of intersection with $S$. Thus the images of the $K$ arcs which are parallel to $\alpha_{k}$ (or $\alpha_{k+1}$ ) are essential arcs in $\pi(S)$. Hence we get an essential rectangle of length $K-1 \geq P(S)$ with respect to the 3-manifold $\pi(X)$ and surface $\pi(S)$, which contradicts Lemma 2.3.

Proof of Theorem 1.2 and Theorem 1.3. We will prove that the surface $T$ constructed at the beginning of this section is $\pi_{1}$-injective in $X(\mu)$ if both $\Delta(\mu, s)$ and $K$ are large.

Suppose not, then there exists a closed essential curve $l$ in $T$ contractible in $X(\mu)$. Hence for any $i \geq 1$ there is an immersion $j: P \rightarrow X$, where $P$ is a planar surface with $k+1$ boundary components, $\partial P=\bigcup_{i=0}^{k} p_{i}, j\left(p_{0}\right)=l$ and $j\left(p_{i}\right)$ is an immersed curve of slope $\mu$ in $\partial X$. We assume that $l$ has been homotoped to have the fewest points of intersection with $S$ and $k(\geq 1)$ is the minimal number for all such planar surfaces. The case where $k=0$ follows from Lemma 3.1. Now $j^{-1}(S)$ is a union of disjoint simple arcs or simple closed curves in $P$ because $S$ is embedded. By the same argument 
as before we can assume that there are no trivial circles in $P$.

Claim. There are no $\partial$-parallel $\operatorname{arcs}$ in $P$ with both endpoints on the same $p_{i}$ for any $i \geq 1$.

Proof of the Claim. Suppose there are such arcs. We choose an outermost one, say $\gamma$, which together with a subarc $\gamma^{\prime}$ of $p_{i}$ bounds a bigon in $P$. Hence $j(\gamma)$ can be homotoped rel boundary into $\partial X$. Since $S$ is $\partial$-incompressible, both endpoints of $j\left(\gamma^{\prime}\right)$ must lie in the same component of $\partial S$ and $j\left(\operatorname{int}\left(\gamma^{\prime}\right)\right) \cap$ $\partial S=\emptyset$ (because $\gamma$ is outermost). So we can homotope $p_{i}$ to get fewer points of intersection with $\partial S$, which contradicts our assumption.

Let $\mathcal{B}$ be the subset of $j^{-1}(S)$ consisting of arcs with at least one endpoint on $p_{i}$ for some $i \geq 1$. Since $j\left(p_{i}\right)$ is a curve of slope $\mu$ in $\partial X$ for $i \geq 1, j\left(p_{i}\right)$ intersects $S$ in at least $2 \Delta(\mu, s)$ points (we have assumed that $S$ has two boundary components). Hence $|\mathcal{B}| \geq k \Delta(\mu, s)$. By an Euler Characteristic argument, the maximal number of non-parallel arcs in $P$ is $3 k-3$ if $k>1$, and 1 if $k=1$. So, if $|\mathcal{B}| \geq k \Delta(\mu, s) \geq 3 k N$, there are at least $N+1 \operatorname{arcs}$ in $\mathcal{B}$ which are parallel to each other. Let $\delta_{0}, \delta_{1}, \ldots, \delta_{N}$ be the $N+1$ parallel arcs.

Case 1. The $N+1$ parallel arcs have endpoints on $p_{i}$ and $p_{j}$ with both $i, j \geq 1$.

Recall that by our construction $j\left(p_{i}\right) \subset \partial X$ if $i \geq 1$.

Suppose $j\left(\delta_{i}\right)$ is a $\partial$-parallel arc in $S$ for some $i$. Then we can homotope $j\left(\delta_{i}\right)$ to $\partial X$, then cut along $\delta_{i}$ to get a map of a planar surface with fewer boundary components, which contradicts our assumption.

Therefore $j\left(\delta_{i}\right)$ is an essential arc for every $i$ and $\delta_{0}, \delta_{1}, \ldots, \delta_{N}$ form an essential rectangle of length $N$. By Lemma 2.3, if $N \geq P(S)$, no such essential rectangle exists.

Case 2. Each of the $N+1$ parallel arcs has one endpoint on $p_{0}$ and the other endpoint on $p_{i}$ for some $i \geq 1$.

As in the proof of Lemma 3.1, we divide $p_{0}$ into segments $\alpha_{1}$, $\beta_{1}, \ldots, \alpha_{q}, \beta_{q}$, where $p_{0}=\bigcup_{i=1}^{q}\left(\alpha_{i} \cup \beta_{i}\right)$ and each $j\left(\beta_{i}\right)$ is a subarc of $l$ lying entirely in the long annulus, each $j\left(\alpha_{i}\right)$ is a subarc of $l$ lying entirely in the surface $S^{\prime}$. We call the $\alpha_{i}$ 's $\alpha$-arcs and the $\beta_{i}$ 's $\beta$-arcs.

$\delta_{0} \cup \delta_{N}$ together with a subarc $\sigma$ of $p_{0}$ and a subarc $\rho$ of $p_{i}$ form a quadrilateral $Q$ in $P$ (see Figure 2 (a)). By the claim, there are no arcs in $Q$ with both endpoints on $\rho$. So the $\operatorname{arcs}$ of $j^{-1}(S)-\mathcal{B}$ in $Q$ are arcs with both endpoints on $\sigma$. By the claim 1 in Lemma 3.1, there are no $\operatorname{arcs}$ in $Q$ 


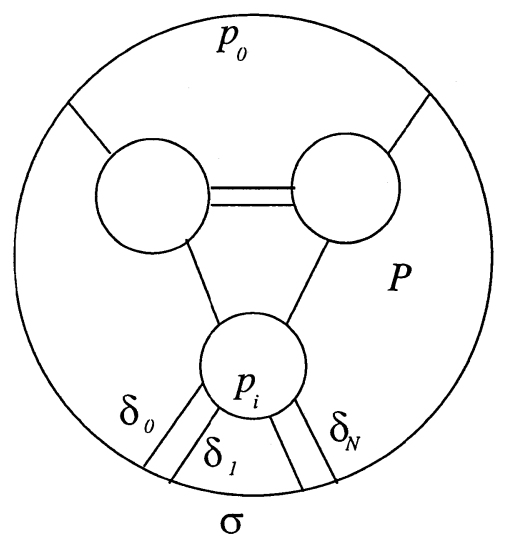

(a)

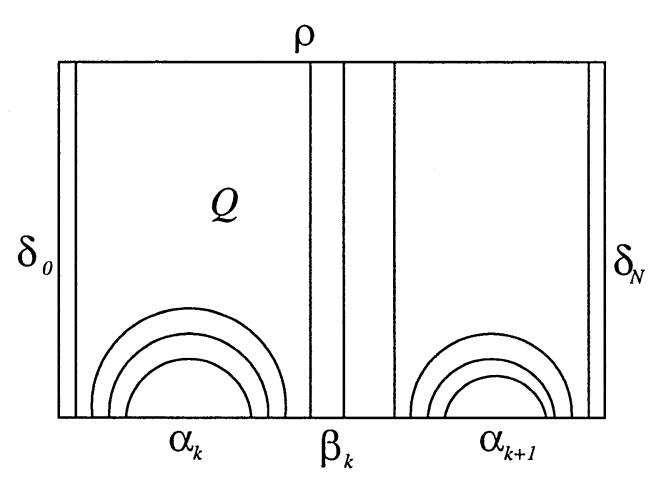

(b)

Figure 2.

with both endpoints on the same $\beta$-arc. As in the proof of Lemma 3.1, we can assume that there is no long $\operatorname{arc}$ in $Q$, i.e. no arc cutting off a bigon in $Q$ which contains at least two $\alpha$-arcs.

Next we choose $K$ and $N$ such that $2 K \geq 3 P(S)+1$ and $N+1 \geq$ $2 K+2 P(S)+1$. Since each $\beta$-arc contains exactly $2 K$ endpoints of arcs in $j^{-1}(S)$, there is at least one $\alpha$-arc in $Q$.

Suppose there are at least two $\alpha$-arcs, say $\alpha_{k}$ and $\alpha_{k+1}$, in $Q$ (by choosing $N$ larger, we can always ensure that). Then all the arcs with one endpoint on $\beta_{k}$ are contained in $Q$. As in the proof of Lemma 3.1, there are at most $P(S)$ arcs parallel to $\alpha_{k}$ or $\alpha_{k+1}$, otherwise we have an essential rectangle of length $P(S)$. So there are at least $2 K-2 P(S) \geq P(S)+1$ arcs with one endpoint on $\beta_{k}$ and the other endpoint on $\rho$, as shown in Figure 2 (b). If the images of these $P(S)+1$ arcs under the map $\pi \circ j$ are not trivial in $\pi(S)$, we get an essential rectangle of length $P(S)$, which contradicts Lemma 2.3. Therefore we assume that they are trivial $\operatorname{arcs}$ in $\pi(S)$.

Since $N+1 \geq 2 K+2 P(S)+1$, we have at least $P(S)+1$ parallel arcs in $\mathcal{B}$ with one endpoint on $\beta_{k+1}$ (or $\beta_{k-1}$ ). Again we assume the image of these parallel arcs under $\pi \circ j$ are trivial in $\pi(S)$; otherwise it contradicts Lemma 2.3.

Let $\bigcup_{i=1}^{2 K} l_{i}=T \cap S$, where each $l_{i}$ is a simple closed curve. Then by 
our construction of $T$ and assumption on $l$, the intersection of each $\beta_{i}$ with $T \cap S$ appears either in the order (with respect of a certain orientation of $l$ ) $l_{1}, l_{2}, \ldots, l_{2 K}$ or in the order $l_{2 K}, l_{2 K-1}, \ldots, l_{1}$. An arc that has one endpoint in the central portion of $\beta_{k}$ or $\beta_{k+1}$ (or $\beta_{k-1}$ ) must have the other endpoint on $\rho$, and hence is one of the $N+1$ parallel arcs that we considered above (see Figure $2(b))$. The reason is that we cannot have too many arcs parallel to the two $\alpha$-arcs adjacent to this $\beta$-arc, otherwise we will get a long essential rectangle. So it is easy to see that there must be an arc $\delta_{i}$ with $\delta_{i} \cap \beta_{k}=a$ and an $\operatorname{arc} \delta_{j}$ with $\delta_{j} \cap \beta_{k \pm 1}=b$ such that $j(a)$ and $j(b)$ lie on the same simple closed curve component of $T \cap S$. Since by our assumption $\pi \circ j\left(\delta_{i}\right)$ and $\pi \circ j\left(\delta_{j}\right)$ are trivial arcs in $\pi(S)$, we can homotope $j\left(\delta_{i}\right)$, moving $j(a)$ along the simple closed curve component of $T \cap S$ to $j(b)$ and closing up $j\left(\delta_{i}\right)$ and $j\left(\delta_{j}\right)$, as shown in Figure 3, to get an immersed annulus in $X$.

One boundary component, say $\sigma^{\prime}$, of this immersed annulus is mapped into $T$ and the other boundary component is mapped into $\partial X$ with slope different from that of $\partial S$. Notice that there is exactly one $\alpha$-arc between $\delta_{i}$ and $\delta_{j}$ in $Q$. If $\sigma^{\prime}$ is mapped to a trivial curve in $T$, then the $\alpha$-arc between $\delta_{i}$ and $\delta_{j}$ must be a $\partial$-parallel arc in $S^{\prime}$, and we can homotope $l$ to get fewer points of intersection with $S$, which contradicts our assumptions. So $\sigma^{\prime}$ is a non-trivial curve in $T$. Now the simple closed curve component of $T \cap S$ containing $j(a)$ and $j(b)$ together with a boundary component of $\partial S$ bounds another annulus in $S$, and the intersections of the two annuli are vertical arcs in both of them. Therefore we get two elements in $\pi_{1}(T)$ simultaneously homotopic to two curves in $\partial X$ of different slopes. Since $T$ is $\pi_{1}$-injective in $X$ and clearly $T$ is not peripheral, $\mathbb{Z} \oplus \mathbb{Z}$ is a non-peripheral subgroup of $\pi_{1}(X)$. This contradicts the hypothesis that $X$ is hyperbolic.

So, as long as $2 K \geq 3 P(S)+1$ and $N+1 \geq 2 K+2 P(S)+1$ (i.e. $N \geq 5 P(S)+1), T$ is $\pi_{1}$-injective in $X(\mu)$. Recall that we have chosen $k \Delta(\mu, s) \geq 3 k N$ to get $N+1$ parallel arcs. Hence it suffices that $\Delta(\mu, s) \geq$ $3 N \geq 15 P(S)+3$.

If $Q$ contains exactly one $\alpha$-arc, then as in the proof of Lemma 3.1, there are at most $P(S)$ arcs parallel to this $\alpha$-arc. Since $N \geq 2 K+2 P(S)$, incident to each $\beta$-arc that is adjacent to this $\alpha$-arc, there are at least $2 P(S)$ arcs belonging to the set of the $N+1$ parallel arcs that we considered above. Now the proof is as earlier.

Remarks. If $S$ has more that two boundary components, then $|\mathcal{B}| \geq$ $\frac{b}{2} k \Delta(\mu, s)$. In this case, there are $\frac{b}{2}$ long annuli and we need $j(a)$ and $j(b)$ to be on the same long annulus. Hence the factor $b$ will be canceled and we 


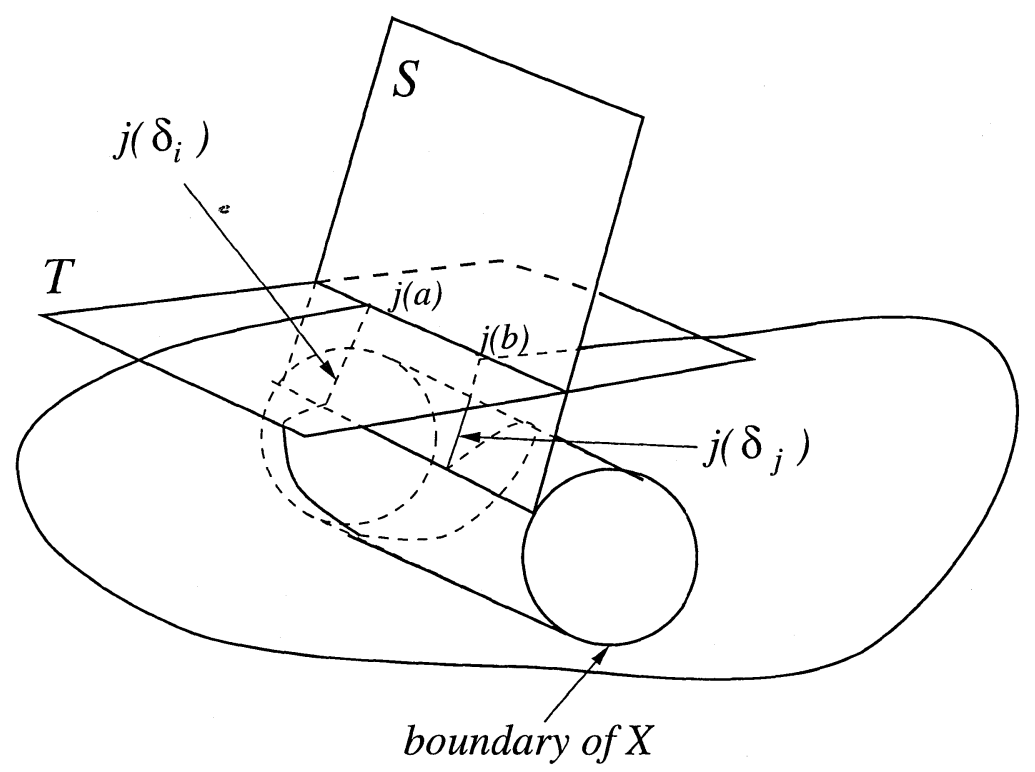

Figure 3.

get a bound for $\Delta(\mu, s)$ that is a linear function of $g$ and $b$.

\section{References.}

[1] M. Baker, On boundary slopes of immersed incompressible surfaces, Ann. Inst. Fourier (Grenoble), 46 (1996), 1443-1449.

[2] D. Cooper and D.D. Long, Virtually Haken surgery on knots, Preprint.

[3] D. Cooper and D.D. Long, Some surface subgroups survive surgery, Preprint.

[4] D. Cooper, D.D. Long and A.W. Reid, Essential closed surfaces in bounded 3-manifolds, J.Amer.Math.Soc., 10 (1997), 553-564.

[5] M. Culler, C.McA. Gordon, J. Lueke and P.B. Shalen, Dehn surgery on knots, Ann. of Math., 125 (1987), 237-300.

[6] M. Culler and P.B. Shalen, Bounded, separating, incompressible surfaces in knot manifolds, Inven. Math., 75 (1984) 537-545. 
[7] B. Freedman and M.H. Freedman, Kneser-Haken finiteness for bounded 3-manifolds locally free groups, and cyclic covers, Topology, 37(1) (1998), 133-147.

[8] A. Hatcher, On the boundary curves of incompressible surfaces, Pacific J. Math., 99 (1982), 373-377.

[9] W. Jaco, Lectures on Three-Manifold Topology, CBMS Regional Conference Series in Mathematics, 43 (1977).

[10] W. Jaco and P.B. Shalen, Seifert fibered spaces in 3-manifolds, Mem. Amer. Math. Soc., 21(220) (1979).

[11] K. Johannson, Homotopy equivalences of 3-manifolds with boundary, Lecture Notes in Mathematics, 761, Springer, 1979.

[12] W. Menasco, Closed incompressible surfaces in alternating knot and link complements, Topology, 23(1) (1984), 37-44.

[13] U. Oertel, Boundaries of injective surfaces, Topology Appl., 78(3) (1997), 215-234.

[14] W.P. Thurston, Three-dimensional manifolds, Kleinian groups and hyperbolic geometry, Bull. Amer. Math. Soc., 6(3) (1982), 357-381.

[15] F. Waldhausen, On irreducible 3-manifolds which are sufficiently large, Ann. of Math., 87 (1968), 56-88.

Department of Mathematics

OKLAHOMA STATE UNIVERSiTy

STILLWATER, OK 74078

Received September 10, 1999. 